\title{
Data Collection: Poultry Integrations ${ }^{1}$
}

\section{G. D. Butcher, DVM, Ph.D. and A. H. Nilipour²}

The poultry industry is continuing to expand rapidly throughout many regions of the world. World production of chicken and eggs is twice the level of only two decades ago. The genetic potential of the broiler, breeder and egg-type layer has changed dramatically, resulting in a higher producing but more fragile chicken. Thus, the commercial chicken of today requires improved management practices. In today's highly sophisticated industry, data collection practices implemented by a company determine how data are interpreted and whether conclusions are correct or erroneous. In the field, many potential opportunities to evaluate production are encountered daily. Often these opportunities are lost, as data collection systems are not organized and data generated cannot be evaluated and applied.

A poultry producer must have a complete and accessible data bank when production problems are encountered to facilitate a quick and successful resolution. Because live animals are involved, timely and accurate decisions play a major role in preventing greater losses due to disease outbreaks, feed toxicity, poor management, inadequate nutrition, ill-advised health programs, substandard housing, etc. The factor to consider is that most production problems can easily be resolved once the problems are clearly defined. With a comprehensive database, this investigative process is greatly facilitated.

In this article, a point rarely discussed in industry magazines and scientific journals will be defined: How to collect data to permit competent and timely decision making.

\section{The Data Collection}

In a recent survey it was revealed that successful companies (Fortune 500) with high net earnings were those that paid close attention to collecting and analyzing information. These companies invested to update the methods of information processing by installing modern computers and keeping in touch with the latest technologies. Currently, there are companies solely dedicated to developing software to permit better collection and assimilation of data. These companies provide a valuable service. However, purchase of these expensive services is not necessary for most companies. Simply with a basic knowledge of computers and access to most current spreadsheet programs such as Excel or Lotus, a tailored program for most companies can be designed. After experimenting with the program, adaptations can be incorporated to accommodate the specific needs of your company.

1. This document is VM140, one of a series of the Veterinary Medicine-Large Animal Clinical Sciences Department, Florida Cooperative Extension Service, Institute of Food and Agricultural Sciences, University of Florida. Original publication date November 2005. Visit the EDIS Web Site at http://edis.ifas.ufl.edu.

2. Gary D. Butcher, DVM, Ph.D., Diplomate, American College of Poultry Veterinarians, University of Florida College of Veterinary Medicine, Gainesville, FL., Amir H. Nilipour, PhD, Director of Investigation and Quality Assurance, Grupo Melo, S.A., Panama, Republic of Panama 


\section{Compare Results to Established Standards}

After designing the system for information collection and assimilation, it is important to compare the results generated with previously established standards or goals. It would not make any sense to generate information and be unable to interpret its significance for lack of a set of standards. Develop internal standards to fit local needs and specifications, or consult with commercial manuals provided by primary breeders with the specific genetic potential for each line. These guides should be looked upon as reference points. Local conditions in some areas may make it difficult to achieve the suggested performance. Therefore, specific standards for your company need to be formulated.

\section{Graph the Information}

Performance information needs to be collected on a regular basis. Collection frequency depends on the specific parameters measured. The data could be collected at hourly, daily, weekly, monthly or yearly intervals, depending on the parameter evaluated and the available labor. Whatever is chosen, consistency must be maintained. Following data collection, the information needs to be assimilated in a manner that facilitates analysis. One method is to plot the data to make it easier to visualize the tendencies. Looking at a table of numbers in a dry form is of limited value and even might be confusing. To illustrate this point, review the following broiler data for the last three years in two forms, tables and graph formats. Please look at the table first and then at the graph to determine which one was easier to interpret.

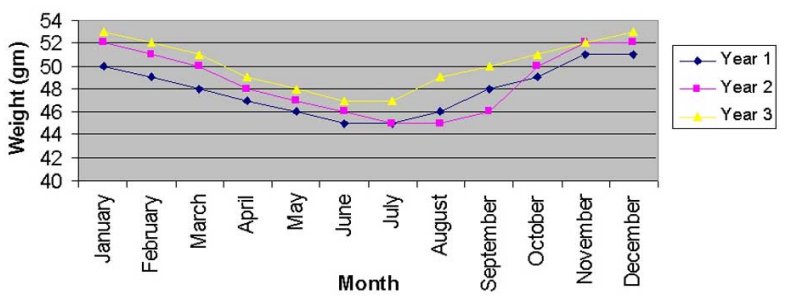

Figure 1. Monthly average weight gains of broilers for 3 years

\section{Take Adequate Sample Size}

Table 1. Monthly average weight gains of broilers for 3 years

\begin{tabular}{||c|c|c|c||}
\hline \multirow{2}{*}{ Month } & \multicolumn{3}{|c||}{ Weight (in grams) } \\
\cline { 2 - 4 } & Year 1 & Year 2 & Year 3 \\
\hline January & 50 & 52 & 53 \\
\hline February & 49 & 51 & 52 \\
\hline March & 48 & 50 & 51 \\
\hline April & 47 & 48 & 49 \\
\hline May & 46 & 47 & 48 \\
\hline June & 45 & 46 & 47 \\
\hline July & 45 & 45 & 47 \\
\hline August & 46 & 45 & 49 \\
\hline September & 48 & 46 & 50 \\
\hline October & 49 & 50 & 51 \\
\hline November & 51 & 52 & 52 \\
\hline December & 51 & 52 & 53 \\
\hline \hline
\end{tabular}

Routine collection of samples to monitor performance tendencies at the farm is essential, even when production performance is "normal." Some of the more common variables that need to be monitored include:

Body weights: For all types of poultry, including broilers, breeders and layers, achieving and maintaining optimum body weight can have a significant economic impact. Taking an adequate sample of birds can assure that the body weight data obtained is representative of the flock. For the breeders and layers, it is normally recommended to individually weigh 3 to $5 \%$ of the birds. For the broilers, weigh 25 to 50 birds, depending on the size of the broiler house. When catching the birds, it is important to encircle them, causing the least amount of stress, and catch them in various parts of the house to obtain a representative sample of the house population. All the birds collected must be weighed to prevent slanting the results. On many occasions farmers have a tendency to weigh the better looking birds, and let the runts or smaller ones escape.

Through 3 to 4 weeks of age, it is suggested to utilize digital scales for more accuracy. Avoid using scales based on pounds; instead use the gram-based scales.

Ingredients and feed samples: Feed analysis, contrary to the weighing of birds, is less commonly practiced. Usually feed analyses are done only when 
there is a performance problem that could be associated with feed. It is a wise management practice to perform proximal analysis for all batches of ingredients arriving at the feed mill. Normally, 10 to 12 samples are taken from a batch at various points and depths, mixed and from these samples, 2 to 4 sub-samples are taken to run the tests.

The same procedure must be practiced for the mycotoxin presence in the raw materials and feeds. It is recommended to sample and store 2 to 4 fifty-gram feed samples of all the feed batches that arrive to a farm. Those should be held at the location for about two weeks after collection. If you suspect feed problems have caused lower than optimum performance, these feed samples could be analyzed for abnormalities.

Feed mixing: How a feed is mixed and the uniformity of the vital micro-nutrients such as vitamins, minerals or growth promoters can markedly change performance. At least once a week, 10 samples should be taken from each of the mixers and the Coefficient of Variation calculated. The best $\mathrm{CV} \%$ is obtained when it is less than 1.05 . If the $\mathrm{CV}$ is above $10 \%$ corrective actions must be taken to resolve the problem.

Tissue and serum samples: This is likely the most misunderstood part of sample collecting. On visits to poultry operations in various countries, it was recognized that good intentions on the part of the technical service personnel may result in devastating losses. On many occasions there is no logic for the way samples are collected and why. Many producers and technical personnel collect samples only to get done with the responsibility of submitting the samples to laboratories for serology or histopathology studies. When samples are submitted to the laboratory, in many cases, results generated will be of limited value due to errors in sampling procedures. It is wise to discuss sample collection with a qualified poultry veterinarian prior to submission if there are any questions. To elaborate, procedures to consider when collecting and submitting quality samples to the diagnostic laboratory include:
- Identify the sample properly. The sample must be properly coded to prevent mix up and to ensure that results can be retrieved for future reference.

- Provide farm history, such as management practices, age, sex and species of bird, environmental conditions, mortality patterns, etc.

- State the reason the sample was submitted.

- Provide the proper quantity of samples for the specific tests.

- Transport samples carefully. Samples should be properly packed and mailed by a courier to assure they arrive in good condition.

- Provide a sample permit if one is required. Many countries do not allow entry of sera or tissues without a permit. The requirements of the permit should be acknowledged and a copy should be submitted along with the samples.

- Collect the sample at the right time.

A comprehensive understanding of the uses of serology in commercial poultry flocks is essential to ensure that samples are collected properly. In summary, a distinction must be made between serologic testing for diagnostics and serologic testing for flock monitoring. Diagnostic testing attempts to relate a disease condition to rising antibody titers. Flock monitoring is performed to evaluate a poultry health program. Diagnostic serology and monitoring serology are similar in that results of both are expressed as antibody titers and titers are determined by identical laboratory techniques. When samples are submitted for serologic testing, the testing should be performed in the same laboratory, by the same test system, and by the same laboratory technician.

When a chicken is vaccinated or exposed to a disease, it responds by producing antibodies against a specific disease organism. The level of antibodies present in the blood are measured and expressed as titer values.

Antibody levels in the blood are constantly changing (i.e., increasing or decreasing). Conclusions can be made for diagnostic purposes 
only if we know which direction the titers are moving. To determine which direction the antibody titers are going, we must compare two sets of serum samples taken over a 3- to 4-week time span. The first sample is taken at the onset of the disease and the second approximately 4 weeks later. If the titers are rising, this indicates recent exposure. If titers are decreasing, no recent exposure has occurred.

Some of the things flock monitoring can help determine include:

- The effectiveness of the pullet vaccination program;

-Whether boosting of layers/breeders is needed during the production cycle;

- Maternal antibody titer levels;

- A plan for vaccination of chicks;

- Efficacy of vaccine administration;

- Whether birds have been exposed to a disease to which they have not been vaccinated.

When developing a monitoring program, remember that collecting too many samples can result in confusion, while too few samples may not provide enough information. An organized monitoring schedule should be developed so valid interpretations of the poultry health program can be made.

The following are guidelines to follow when collecting tissues to ensure high quality tissue samples are submitted for study:

- Submit formalin fixed tissues only from freshly killed (deceased) birds.

- Use at least 10 times the volume of $10 \%$ neutral buffered formalin to the volume of tissue taken for histopathology.

- Use only containers with wide openings. It is safest to place the formalin container in a sturdy, sealable, plastic bag when shipping.

- Do not freeze tissues before or after fixation.
- Ensure that tissues are no more than 1/4 inch $(0.5 \mathrm{~cm})$ thick.

- Trim extraneous tissue from the specimens.

- Open all hollow organs (including trachea, GI tract, bursa of Fabricius, cloaca and uterus) prior to fixation. One-half-inch-long to one-inch-long segments are best.

- Be careful not to touch the inside surfaces of hollow organs if submitting the tissue. Excess ingesta or blood can be rinsed in another container of formalin (or saline).

- Avoid squeezing or distorting tissue with forceps.

- Cut the spleen, the heart, and the brain in half to allow contact with fixative.

- Strip bone of skin and muscle, and incise to allow fixative to enter the core.

- Cover floating tissue with gauze or paper towel.

- Use tissue cassettes when submitting small pieces of tissue such as air sacs or peripheral nerves.

- Make sure containers and/or tissues are labeled and properly sealed to prevent leakage during transport. Receipt of poor quality samples will greatly decrease the ability to provide an accurate diagnosis.

\section{Keep It Simple}

Raising chickens is not a complicated job, but can become one due to lack of adequate knowledge and experience. At all times, the philosophy of keeping the routines as simple as possible by following certain basic rules of nature, statistics and by implementing a solid biosecurity and health program must be adhered to. Data and sample collection are parts of the business that must be performed properly if the company is to succeed. Any problem that arises is a puzzle at first, but analysis of the data without panicking will permit resolutions of most problems in an effective and rapid manner. 